\title{
Slavnov-Taylor Identity for the Effective Field Theory of the Color Glass Condensate
}

\section{Binosi}

European Centre for Theoretical Studies in Nuclear Physics and Related Areas (ECT*) and Fondazione Bruno Kessler, Trento, Italy

E-mail: binosidectstar.eu

\section{A. Quadri*}

Univ. degli Studi di Milano \& INFN, Sezione di Milano

via Celoria 16, I-20133 Milano, Italy

E-mail: andrea.quadri@mi.infn.it

\section{N. Triantafyllopoulos}

European Centre for Theoretical Studies in Nuclear Physics and Related Areas (ECT*) and Fondazione Bruno Kessler, Trento, Italy

E-mail: triantadectstar.eu

\begin{abstract}
We show that a powerful Slavnov-Taylor (ST) identity exists for the Effective Field Theory (EFT) of the Color Glass Condensate (CGC), allowing to control by purely algebraic means the full dependence on the background fields of the fast gluon modes, as well as the correlators of the quantum fluctuations of the classical gluon source. We use this formalism to study the change of the background fast modes (in the Coulomb gauge), induced by the quantum corrections of the semi-fast gluons. We establish the evolution equation for the EFT of the CGC, which points towards an algebraic derivation of the JIMWLK equation. Being based on symmetry-arguments only, the approach can be used to extend the analysis to arbitrary gauges and to higher orders in the perturbation expansion of the EFT.
\end{abstract}

The European Physical Society Conference on High Energy Physics -EPS-HEP2013

18-24 July 2013

Stockholm, Sweden

\footnotetext{
*Speaker.
} 


\section{Introduction}

At high energies and high gluon density the regime of gluon saturation in the small $x$ region (where $x$ denotes the longitudinal momentum fraction of the parton) is well described by the effective field theory (EFT) of the Color Glass Condensate (CGC) [1].

A well-known application of the CGC formalism is the study of heavy ion collisions [1]-[4], where gluon saturation plays a prominent role in the determination of the initial wavefunction of the projectile and target nuclei as well as in the early phases of the collision.

The EFT of the CGC allows to derive an evolution equation for the wavefunction of a hadron (or a nucleus) to arbitrarily small $x$ values. This evolution is controlled by a renormalization group (RG) equation, the JIMWLK equation [5], that makes it possible to resum the large logarithmic corrections induced by the radiation of quantum gluons in a dense environment.

In the CGC picture, the fast colour sources $\rho$ are described by classical configurations associated with a probability distribution $W_{\Lambda}[\rho]$ at a given energy scale $\Lambda$. The colour sources generate through the Yang-Mills equations of motion a set of classical (background) gauge fields, describing the fast gluon modes in the infinite momentum frame, i.e. those wtih momentum component $p^{+}$ above $\Lambda$.

Quantum gluons are then radiated off the background fast configurations. In the EFT approach, one needs to integrate the semifast gluon modes with momentum between $b \Lambda$ and $\Lambda$, where $b$ is a small parameter such that $\alpha_{s} \ln \frac{1}{b} \ll 1$ (so that a weak coupling expansion is reliable, despite the fact that the classical field configurations are strong, being of the order $\sim \frac{1}{g}$, and will be resummed).

Then one finds that, in the leading logarithmic approximation, the radiative corrections amount to the redefinition of the probability distribution $W_{b \Lambda}[\rho]$, which now describes the CGC at the scale $b \Lambda$. The classical Yang-Mills equations of motion, relating the classical fast gluons to the colour sources $\rho$ at the new scale, are not modified by the quantum evolution.

The flow from the original probability distribution $W_{\Lambda}$ to the new one $W_{b \Lambda}$ is generated by an effective Hamiltonian [5, 6]. The resulting functional Fokker-Planck equation for the weight function $W_{\Lambda}[\rho]$ provides the appropriate mathematical description for the one-step quantum evolution in the CGC framework.

It turns out that these results can be traced back to the symmetry content of QCD (in the presence of external gluon field configurations), namely to the Slavnov-Taylor (ST) identity of the theory. In particular, the deformation of the background fast gluon fields, induced by the integration of the semifast modes, is controlled by the ST identity. This result extends the one given in [7]-[9], where it is shown that the quantum deformation of the background field can be obtained through a canonical transformation whose form is dictated by the ST identity. Moreover, the ST identity also controls the quantum deformation of the Yang-Mills equations of motion for the fast background gluons.

By exploiting these constraints it is possible to obtain an evolution equation of the effective action (after integration of the semifast modes) with respect to (w.r.t.) the parameter $b$. This holds in a rather general setting and points towards a purely algebraic derivation of the JIMWLK evolution equation. 


\section{The classical theory of the CGC}

The Yang-Mills (YM) action in the presence of a colour source $\rho$ is

$$
S_{\mathrm{CGC}}[A, \rho]=S_{\mathrm{YM}}[A]+S_{W}[A, \rho] .
$$

The conventional Yang-Mills action is given by

$$
S_{\mathrm{YM}}=-\frac{1}{4 g^{2}} \int d^{4} x F_{a \mu v} F_{a}^{\mu v}
$$

where $F_{a \mu \nu}$ denotes the YM field strength $F_{a \mu \nu}=\partial_{\mu} A_{a v}-\partial_{v} A_{a \mu}+f_{a b c} A_{b \mu} A_{c v}$. On the other hand, $S_{W}[A, \rho]$ describes the gauge-invariant interaction between the colour sources $\rho$ and the gauge field $A$ built from the contour temporal Wilson line $W_{C}(\vec{x})$, namely

$$
S_{W}[A, \rho]=\frac{i}{g} \int d^{3} \vec{x} \operatorname{Tr}\left[\rho(\vec{x}) W_{C}(\vec{x})\right]
$$

We use light-cone coordinates $x^{\mu}=\left(x^{+}, x^{-}, \mathbf{x}\right)$ with $x^{ \pm}=\left(x^{0} \pm x^{3}\right) / \sqrt{2}, \mathbf{x}=\left(x^{1}, x^{2}\right)$ and $\vec{x}=\left(x^{-}, \mathbf{x}\right)$. The contour $C$ is obtained in the limit $x_{0}^{+} \rightarrow-\infty$ and $x_{f}^{+} \rightarrow+\infty$ of the Schwinger-Keldysh contour in the complex time plane $C_{+} \cup C_{-}$, where $C_{+}$is the path along the real time axis from $x_{0}^{+}$to $x_{f}^{+}$ and $C_{-}$is the set of points with a small imaginary part $z=x^{+}-i \eta$, with $\eta \rightarrow 0_{+}$and $x^{+}$running backwards from $x_{f}^{+}$to $x_{0}^{+}$.

At leading order $S_{W}$ yields a coupling $\rho^{a}(\vec{x}) A_{a}^{-}(\vec{x})$, where $\rho^{a}$ is the plus component of the fast color current (and the only non-vanishing). Notice that $\rho^{a}$ is $x^{+}$-independent, i.e. it represents fast static color charges.

The only component of the gauge field that couples to the color sources is $A^{-}$. Its equation of motion $\frac{\delta S}{\delta A^{-}}=0$ yields in matrix notation

$$
D_{v}[A] F^{v \mu}=\delta^{\mu+} W_{x^{+},-\infty}(x) \rho(\vec{x}) W_{x^{+},-\infty}^{\dagger}(x)
$$

where we have set $A_{\mu}=A_{a \mu} T_{a}, \rho=\rho_{a} T_{a}$ and $D_{\mu} \Phi=\partial_{\mu} \Phi-i[A, \Phi]$ for a generic field $\Phi=\Phi_{a} T_{a}$ in the $\mathrm{SU}(\mathrm{N})$ representation spanned by the generators $T_{a}$. By keeping the leading $A^{-}$-independent term in the R.H.S. of eq.(2.4), one can choose a solution where $A^{i}$ is pure gauge. In the Coulomb gauge $\partial_{l} A^{l}=0$ the only non-vanishing component is $A^{+}$and eq.(2.4) then reduces to the Poisson equation

$$
-\nabla_{\mathbf{x}}^{2} A^{+}(\vec{x})=\rho(\vec{x})
$$

which fixes the gluon field configuration in the presence of the fast color charges $\rho$.

\section{BRST Symmetry and Gluon Layers}

In the CGC approach one would like to compute the radiative corrections induced by the exchange of semifast quantum gluons with the classical solution $A^{+}$fulfilling eq.(2.5). For that purpose the gluon field $A$ is split into three components as follows:

$$
A_{\mu}=\delta A_{\mu}+a_{\mu}+\hat{A}_{\mu}
$$


The background field $\hat{A}_{\mu}$ describes the fast configuration fulfilling eq.(2.5) and has support only in the fast region $\left|p^{+}\right| \geq \Lambda$. The semi-fast gluons $a_{\mu}$ have support in the region $b \Lambda<\left|p^{+}\right|<\Lambda$ and have to be integrated out in the one-step quantum evolution. Finally, $\delta A_{\mu}$ are the soft modes at $\left|p^{+}\right| \leq b \Lambda$, so that the full background configuration around which the semifast expansion takes place is $\delta A+\hat{A}$. Notice that this is not a stationary point of the YM action.

In order to carry out the integration over $a_{\mu}$, one needs to fix the gauge. The usual choice is to adopt the light-cone gauge condition $n^{\mu} A_{\mu}=0$, while keeping the Coulomb gauge for $\delta A_{\mu}$.

Once the gauge-fixing is performed, gauge invariance of the classical theory is lost and physical unitarity cancellations are provided by the ST identity, associated with the BRST symmetry of the model. In the CGC approach one has in addition the presence of a background source $\hat{A}_{\mu}$. The latter is controlled by using an extended BRST symmetry and implementing a canonical transformation that fixes uniquely the dependence on the background $\hat{A}_{\mu}$, along the lines of [9]. Consequently one sets $s \hat{A}_{a \mu}=\Omega_{a \mu}, s \Omega_{a \mu}=0$, where $\Omega_{a \mu}$ is the BRST partner of the background. The derivative of the effective action w.r.t $\Omega_{a \mu}$ can be understood as the generating functional of the canonical transformation which controls the dependence on $\hat{A}_{\mu}$. Moreover, the BRST transformation of the full gauge field $A_{\mu}$ is bound to be $s A_{a \mu}=\partial_{\mu} c_{a}+f_{a b c} A_{b \mu} c_{c}$, where $c_{a}$ is the ghost. While the BRST transformation of $A_{a \mu}$ and of $\hat{A}_{a \mu}$ are fixed, there is an ambiguity in the choice of how $\delta A_{a \mu}$ and $a_{a \mu}$ must transform, since only the sum of their BRST transformation is constrained. This ambiguity is resolved by noticing that one wishes to preserve gauge invariance of $S_{W}[\delta A, \rho][6]$, so $\delta A$ must transform as a gauge connection, i.e. $s \delta A_{a \mu}=\partial_{\mu} c_{a}+f_{a b c} \delta A_{b} c_{c}$ and thus finally $s a_{a \mu}=f_{a b c}\left(a_{b \mu}+\hat{A}_{b \mu}\right) c_{c}-\Omega_{a \mu}$.

Also the colour sources $\rho$ have to be split according to $\rho=\delta \rho+\hat{\rho}$, where $\hat{\rho}$ are the classical fast colour sources and $\delta \rho$ are the quantum corrections to the classical approximation. The full source $\rho_{a}$ transforms in the adjoint representation, i.e. $s \rho_{a}=f_{a b c} \rho_{b} c_{c}$. On the other hand, by denoting with $\alpha$ the plus component of the background $\alpha \equiv \hat{A}^{+}$, from eq.(2.5) one gets

$$
\hat{\rho}(\vec{x})=-U(x) \nabla_{\mathbf{x}}^{2} \alpha(\vec{x}) U^{\dagger}(\vec{x}), \quad U^{\dagger}(\vec{x})=\mathrm{P}\left[i \int_{-\infty}^{x^{-}} \mathrm{d} z^{-} \alpha\left(z^{-}, \vec{x}\right)\right]
$$

from which one obtains the BRST transformation of $\hat{\rho}$, induced by the BRST transformation of $\hat{A}$ :

$$
s \hat{\rho}=-\int \mathrm{d}^{4} z \Omega_{a}^{+}(z) \frac{\delta}{\delta \alpha^{a}(z)} \hat{\rho}(\alpha) .
$$

\section{Slavnov-Taylor identity}

The BRST transformation is nonlinear and therefore a suitable set of external sources, known as antifields [10], is required in order to formulate its quantum version in the form of the ST identity. For each quantum field $\varphi$ with a nonlinear BRST variation one introduces a source $\varphi^{*}$ with opposite statistics and ghost charge $\operatorname{gh}\left(\varphi^{*}\right)=-1-\operatorname{gh}(\varphi)$. The BRST variation of $\varphi$ is coupled to $\varphi^{*}$ in the antifield-dependent part $S_{\mathrm{AF}}$ of the action, namely $S_{\mathrm{AF}}=\int \mathrm{d}^{4} x \varphi^{*}(x) s \varphi(x)$. Then the full vertex functional $\Gamma$ of the CGC obeys the ST identity

$$
\begin{gathered}
\mathscr{S}(\Gamma)=\int \mathrm{d}^{4} x\left[\Omega_{a \mu}(x) \frac{\delta \Gamma}{\delta \hat{A}_{a \mu}(x)}+\frac{\delta \Gamma}{\delta\left(\delta A_{\mu}^{* a}(x)\right)} \frac{\delta \Gamma}{\delta\left(\delta A_{\mu}^{a}(x)\right)}+\frac{\delta \Gamma}{\delta a_{\mu}^{* a}(x)} \frac{\delta \Gamma}{\delta a_{\mu}^{a}(x)}+\frac{\delta \Gamma}{\delta c_{a}^{*}(x)} \frac{\delta \Gamma}{\delta c_{a}(x)}\right. \\
\left.+\frac{\delta \Gamma}{\delta\left(\delta \rho^{* a}(x)\right)} \frac{\delta \Gamma}{\delta\left(\delta \rho^{a}(x)\right)}+b^{a}(x) \frac{\delta \Gamma}{\delta \bar{c}^{a}(x)}\right]=0,
\end{gathered}
$$


where $b^{a}$ is the usual Nakanishi-Lautrup field implementing the gauge-fixing constraint and $\bar{c}_{a}$ is the antighost.

After the one-step quantum evolution, both the background quantum-splitting in eq.(3.1) and the classical YM equation of motion (2.5) are in general deformed. If this were the case, as for instance if one quantizes YM theory around an instanton background [8], the assumption that the YM equation of motion still holds and that the classical background splitting is still fulfilled would be violated. This in turn would prevent the identification of the momenta of the updated classical probability distribution $W_{b \Lambda}$ (at the energy scale $b \Lambda$ ) with the quantum correlators of the color charge fluctuations $\delta \rho$, and consequently the EFT approach of the CGC could not be pursued in a mathematically consistent fashion.

However it turns out that indeed the ST identity (4.1) takes care of all the magic: it guarantees that the background-quantum splitting still holds in its classical form also after the one-step quantum evolution and moreover that the functional form of the equation of motion for the background remains unaltered. Let us now sketch the proof of both results.

\subsection{Background deformation}

As a consequence of the extended ST identity, it can be proven [7]-[9] that the classical background configuration is deformed as

$$
\hat{A}_{a \mu} \rightarrow \hat{A}_{a \mu}+\Gamma_{\Omega_{a \mu} \delta A_{b v}^{*}} \hat{A}_{b v}+\ldots
$$

where the dots denote higher orders in $\hat{A}$. The deformation function $\Gamma_{\Omega_{a \mu} \delta A_{b v}^{*}} \equiv \frac{\delta^{2} \Gamma}{\delta \Omega_{a \mu} \delta\left(\delta A^{*}\right)_{b v}}$ is in general non-vanishing. However in the light-cone gauge for the semi-fast modes there are no interactions between the source $\Omega$ and the quantum gluons $a$ and thus $\Gamma_{\Omega_{a \mu}} \delta A_{b v}^{*}$ turns out to be zero (even at $\hat{A} \neq 0$ ). Therefore the background (which is kept in the Coulomb gauge) is not deformed. Incidentally we notice that this justifies the expansion around the configuration of the classical background plus $\delta A$ (at fixed $\delta A$ ) although this is not a minimum of the action and therefore one is not carrying out a saddle point approximation.

\subsection{Background equation of motion}

We now take a derivative w.r.t. $\Omega_{a \mu}$ of eq.(4.1) and then consider the sector at $c=\Omega=b=0$. Since $\Gamma_{\Omega_{a \mu}} \delta A_{b v}^{*}$ is zero, we obtain the simpler equation

$$
\frac{\delta \Gamma}{\delta \hat{A}_{\mu}^{a}(x)}=-\int \mathrm{d}^{4} z \frac{\delta^{2} \Gamma}{\delta \Omega_{\mu}^{a}(x) \delta\left(\delta \rho^{* b}(z)\right)} \frac{\delta \Gamma}{\delta\left(\delta \rho^{b}(z)\right)} .
$$

This is the equation of motion for the background which holds when quantum corrections are taken into account. Since $\delta \rho$ does not enter into the gauge-fixing, it follows that the deformation function $\Gamma_{\Omega_{\mu}^{a} \delta \rho_{b}^{*}} \equiv \frac{\delta^{2} \Gamma}{\delta \Omega_{\mu}^{a} \delta \rho_{b}^{*}}$ stays classical, i.e. $\Gamma_{\Omega_{\mu}^{a} \delta \rho_{b}^{*}}=\Gamma_{\Omega_{\mu}^{a}}^{(0)} \delta \rho_{b}^{*}$. In the leading logarithimic approximation only the first term of the BRST transformation of $\hat{\rho}$ in eq.(3.3) is important and one finds $\Gamma_{\Omega_{a}^{\mu}(x) \delta \rho_{b}^{*}(y)}^{(0)}=\nabla_{\mathbf{x}}^{2} \delta^{(4)}(x-y) \delta_{a b}$, i.e. one recovers the classical equation of motion (3.2) when one sets $\alpha=0$ in the Wilson line $U$. 


\section{Evolution equation}

By the above results we know that the dependence on the scale $b$ cannot enter via the deformation function $\Gamma_{\Omega \delta A^{*}}$ and $\Gamma_{\Omega \delta \rho^{*}}$ (since the first is zero and the latter is purely classical). Hence we can write the following RG equation for the theory in the sector $\delta A-\delta \rho$ :

$$
\begin{aligned}
\frac{\partial \Gamma}{\partial b} & =\int \sum_{n=0}^{\infty} \sum_{m=0}^{\infty} \frac{1}{n ! m !} \widehat{A}_{\mu_{1}}^{a_{1}}\left(x_{1}\right) \cdots \widehat{A}_{\mu_{n}}^{a_{n}}\left(x_{n}\right) \\
& \times\left(\widehat{\rho}^{d_{1}}+\Gamma_{\Omega_{\mu_{1}}^{d_{1}} \delta \rho_{e_{1}^{*}}^{*}}^{(0)} \widehat{\rho}^{e_{1}}+\cdots\right)\left(y_{1}\right) \cdots\left(\widehat{\rho}^{d_{m}}+\Gamma_{\Omega_{\mu_{m}}^{d_{m}} \delta \rho_{e_{m}}^{*}}^{(0)} \widehat{\rho}_{v_{m}}^{e_{m}}+\cdots\right)\left(y_{m}\right) \\
& \times\left.\frac{\partial}{\partial b} \frac{\delta^{m+n} \Gamma}{\delta\left(\delta A_{\mu_{1}}^{a_{1}}\left(x_{1}\right)\right) \cdots \delta\left(\delta A_{\mu_{n}}^{a_{n}}\left(x_{n}\right)\right) \delta\left(\delta \rho^{d_{1}}\left(y_{1}\right)\right) \cdots \delta\left(\delta \rho^{d_{m}}\left(y_{m}\right)\right)}\right|_{\delta A=\delta \rho=\widehat{A}=\widehat{\rho}=0}
\end{aligned}
$$

This equation provides the RG evolution equation for the EFT of the CGC in a very general setting. We stress that it is not yet the JIMWLK evolution equation, since one has to identify the correlators of $\delta \rho$ in the effective field theory with the momenta of the weight fucntion $W_{b \Lambda}$ at the new energy scale $b \Lambda$. After this identification, one can derive from eq.(5.1) the functional differential operator controlling the transition from $W_{\Lambda}[\rho]$ to $W_{b \Lambda}[\rho]$.

The ensuing algebraic derivation as well as the details of the analysis sketched here will be presented elsewhere [11].

\section{References}

[1] F. Gelis, E. Iancu, J. Jalilian-Marian and R. Venugopalan, Ann. Rev. Nucl. Part. Sci. 60 (2010) 463 [arXiv:1002.0333 [hep-ph]].

[2] E. Iancu, "QCD in heavy ion collisions," arXiv:1205.0579 [hep-ph].

[3] Y. V. Kovchegov and E. Levin, "Quantum chromodynamics at high energy," Cambridge University Press, 2012.

[4] T. Lappi, Int. J. Mod. Phys. E 20 (2011) 1 [arXiv:1003.1852 [hep-ph]].

[5] J. Jalilian-Marian, A. Kovner, A. Leonidov and H. Weigert, Nucl. Phys. B 504 (1997) 415 [hep-ph/9701284]; J. Jalilian-Marian, A. Kovner, A. Leonidov and H. Weigert, Phys. Rev. D 59 (1998) 014014 [hep-ph/9706377]; E. Iancu, A. Leonidov and L. D. McLerran, Nucl. Phys. A 692 (2001) 583 [hep-ph/0011241]; E. Iancu, A. Leonidov and L. D. McLerran, Phys. Lett. B 510 (2001) 133 [hep-ph/0102009]. H. Weigert, Nucl. Phys. A 703 (2002) 823 [hep-ph/0004044].

[6] Y. Hatta, E. Iancu, L. McLerran, A. Stasto and D. N. Triantafyllopoulos, Nucl. Phys. A 764 (2006) 423 [hep-ph/0504182].

[7] D. Binosi and A. Quadri, Phys. Rev. D 84 (2011) 065017 [arXiv:1106.3240 [hep-th]].

[8] D. Binosi and A. Quadri, Phys. Rev. D 85 (2012) 085020 [arXiv:1201.1807 [hep-th]].

[9] D. Binosi and A. Quadri, Phys. Rev. D 85 (2012) 121702 [arXiv:1203.6637 [hep-th]].

[10] J. Gomis, J. Paris and S. Samuel, Phys. Rept. 259 (1995) 1 [hep-th/9412228].

[11] D. Binosi, A. Quadri, D. Triantafyllopoulos, in preparation. 